Chinese Journal of Organic Chemistry

\title{
一种合成拉坦前列素的新方法
}

\author{
修志明 ${ }^{a}$ 王立成 ${ }^{b}$ 黄梦媛 $^{a}$ 张 鹏 ${ }^{a}$ 郭佑铭 ${ }^{a}$

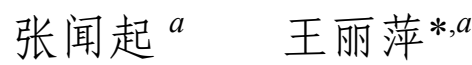 \\ ( ${ }^{a}$ 吉林大学生命科学学院 长春 130012) \\ ( ${ }^{b}$ 吉林大学化学学院 长春 130012)
}

\begin{abstract}
摘要 拉坦前列素是治疗青光眼和高眼压症的首选药物. 现有合成方法存在路线长、收率低、异构体杂质难以分离等 问题. 本研究以苯甲酰基科里内酯为原料, 氧化后与 2-氧代-4-苯丁基膦酸二甲酯通过改良 Horner-Wadsworth-Emmons 反应形成 $\omega$ 侧链, 经还原、脱保护、氢化、四氢吡喃基(THP)保护、还原内酯得到内半缩醛，然后以(4-羧基丁基)三苯 基溴化鏻与六甲基二硅基胺基钠(NaHMDS)形成磷叶立德通过 Wittig 反应形成 $\alpha$ 侧链，经酯化、脱保护基后，合成了拉 坦前列素, 并用正相液相色谱法纯化, 制得终产物, 产物用 ${ }^{1} \mathrm{H} N \mathrm{NM},{ }^{13} \mathrm{C} \mathrm{NMR}, \mathrm{IR}$ 和 HRMS 进行了结构表征. 结果表 明, 拉坦前列素纯度达 $99.91 \%$, 总收率为 $19.2 \%$. 为拉坦前列素的合成提供一种高收率、高纯度、可大批量合成的新方 法.
\end{abstract}

关键词 苯甲酰基科里内酯; 拉坦前列素; 合成; 纯化

\section{A Novel Method for the Synthesis of Latanoprost}

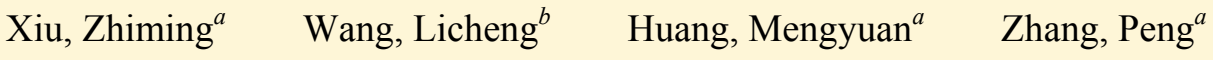 \\ Guo, Youming ${ }^{a} \quad$ Zhang, Wenqi $^{a} \quad$ Wang, Liping ${ }^{*, a}$ \\ ( ${ }^{a}$ School of Life Science, Jilin University, Changchun 130012) \\ ( ${ }^{b}$ School of Chemistry, Jilin University, Changchun 130012)
}

\begin{abstract}
Latanoprost is a kind of $\mathrm{PGF}_{2 \alpha}$ analogues, which is now one of the first-choice drugs in the clinic for open angle glaucoma and ocular hypertension. However, the previous synthetic methods have problems of long steps, low yield and difficult separation of isomeric impurities. Herein, we report a novel method for the synthesis of latanoprost in ten steps. Benzoyl Corey lactone as starting material was oxidized to form corresponding aldehyde by Dess-Martin oxidation. The $\omega$ side chain was bonded by improving Horner-Wadsworth-Emmons reaction with dimethyl(2-oxo-4-phenylbutyl)phosphonate and lithium chloride, and reduced with (-)-diisopinocampheyl chloroborane at $-30{ }^{\circ} \mathrm{C}$ because of its greater selectivity towards the production of desired $S$-isomer (95\%). The deprotection step wherein the protecting group of benzoyl on the hydroxyl group of the cyclopentane ring was removed, was preferably carried out by potassium carbonate in methanol. The hydrogenation of double bond in $\omega$ side chain was carried out by using $5 \%$ palladium-carbon as catalyst. The tetrahydropyranyl (THP) group was used for the protection of the diol with $p$-toluenesulfonic acid as catalyst. Latanoprost lactol was obtained by reducing the lactone with diisobutylaluminum hydride (DIBAL) at $-78{ }^{\circ} \mathrm{C} . \alpha$ side chain of latanoprost was bonded by Wittig reaction with 4-carboxybutyl triphenylphosphoium bromide and NaHMDS forming phosphorus ylide. The carboxylic acid was alkylated with isopropyl iodide in the presence of 1,8-diazabicyclo(5.4.0)undec-7-ene (DBU), and then removed the THP protecting groups with pyridinium 4-toluenesulfonate (PPTS). Latanoprost was separated from two isomers of 15(S)-latanoprost and 5,6-trans-latanoprost after purification by normal-phase high performance liquid chromatography. Thus latanoprost was obtained with high purity of $99.91 \%$ and high overall yield of $19.2 \%$, and the structure was characterized by ${ }^{1} \mathrm{H} N M R,{ }^{13} \mathrm{C} N M R$, IR and HRMS. Compared with the previous routes, the current synthesis is shorter, more practical, and more suitable for large-scale preparation.
\end{abstract}

\footnotetext{
* E-mail: wanglp@jlu.edu.cn; Tel.: 0431-85155348.
}

Received March 27, 2014; revised April 23, 2014; published online May 23, 2014

Project supported by the Natural Science Foundation of Jilin Province (No. 201015171), the Scientific and Technological Planning Poject of Jilin Province (No. 201205017), the Pharmaceutical Industry Development Special Fundation Project of Jilin Province (No. YYZX201150-2), and the Social Development Science and Technology Supported Planning Project of Changchun Technology Bureau (No. 12SF23).

吉林省自然科学基金(No. 201015171)、吉林省科技发展计划(No. 201205017)、吉林省医药产业发展专项资金(No. YYZX201150-2)、长春市科技局社 会发展科技支撑计划(No. 12SF23)资助项目. 
Keywords benzoyl Corey lactone; latanoprost; synthesis; purification

前列腺素(Prostaglandins, 缩写 PGs)是一类重要的 内源性生理活性的物质, 在体内各个组织中广泛存在, 是多种生理过程的重要介质 ${ }^{[1 \sim 6]}$. 前列腺素通常带有一 个环戊烷与两个侧链(简称 $\alpha$ 和 $\omega$ 侧链), 根据五元环的 结构, 天然的前列腺素主要有六类, 如 Scheme 1, 分别 命名为 $\mathrm{PGA}, \mathrm{PGB}, \mathrm{PGC}, \mathrm{PGD}, \mathrm{PGE}$ 和 $\mathrm{PGF}^{[7,8]}$. 其中, $\mathrm{PGF}_{2 \alpha}$ 前列腺素类似物, 由于微量时就可发挥各种生理 作用, 在医药领域已经成为一类重要药物 ${ }^{[9 \sim 12]}$. 拉坦前 列素(latanoprost)是一种 $\mathrm{PGF}_{2 \alpha}$ 类似物, 通过增加房水的 巩膜色素膜外流达到降低眼内压、改变眼的颜色, 因此 作为治疗青光眼及高眼压症的首选药物 ${ }^{[13 \sim 15]}$.<smiles>[R]C1C=CC(=O)C1[R]</smiles><smiles>[R]C1=C([R20])C(=O)CC1</smiles>
PGA<smiles>[R20]C1C(=O)C[C@@H](O)[C@@H]1[R9]</smiles><smiles>[R]C1=CCC(=O)[C@H]1[R]</smiles><smiles>[Pb]</smiles>
III

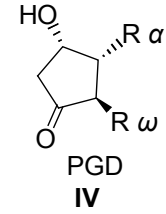

IV<smiles>[R20]C1[C@H]([R20])[C@H](O)C[C@H]1O</smiles>

Scheme 1

拉坦前列素的合成策略中, 主要涉及起始原料的选 择、 $\alpha$ 和 $\omega$ 侧链的选择、保护基的选择、保护和脱保护 步骤及方法的选择等. Obadalova 等 ${ }^{[16]}$ 以 $(1 S, 5 R)-2$-氧杂 双环 [3.2.0]辛-6-烯-3-酮为原料，以叔丁基二甲基硅基 (TBDMS)为保护基，通过 13 步反应立体合成了拉坦前 列素非对应异构体，总收率 $12.3 \%$. 该方法路线较长, 收率低, 且原料和 $\omega$ 侧链难以获得; 科里内酯(Corey Lactone)是合成 $\mathrm{PGF}_{2 \alpha}$ 前列腺素及前列腺素类似物的重

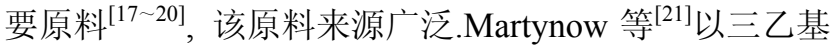
硅基科里内酯为原料，以三乙基硅基(TES)为保护基， 通过 17 步反应合成拉坦前列素，总收率 $16.9 \%$. 该路线 $\alpha$ 和 $\omega$ 两个侧链来源困难, 步骤繁琐, 多步使用柱层析 方法，纯化困难; Resul 等 ${ }^{[22]}$ 报道以苯基苯甲酰基科里内 酯为原料通过 8 步反应合成了拉坦前列素，总收率 5.0\%. 该方法立体选择性差，产生的对映异构体杂质通 过常压柱层析难以分离, 产物纯度低, 收率低.

杂质是该药引发不良反应的重要因素，因此本研究 比较以往合成方法的优劣，避免以上合成方法的不足， 选择以商品化的苯甲酰基科里内酯为原料，设计以 10 步反应合成拉坦前列素的新方法(Scheme 2), 并用正相 液相色谱法纯化，省时、高效，且使拉坦前列素及杂质 得到了良好的分离，提高了总收率和纯度.

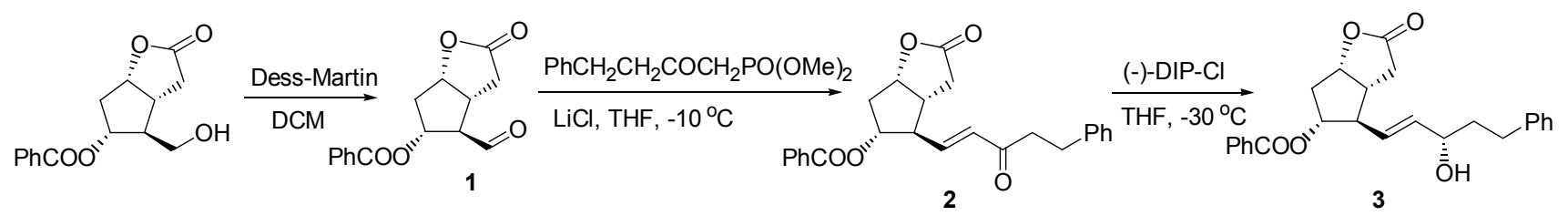

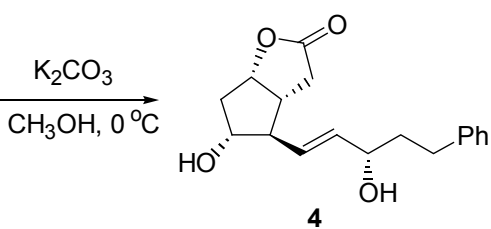
$\underset{\mathrm{EtOH}, 5 \mathrm{MPa}}{5 \% \mathrm{~Pb} / \mathrm{C}, \mathrm{NaNO}_{2}}$<smiles>O=C1C[C@H]2C[C@@H](O)C(=O)C[C@H]2O1</smiles>

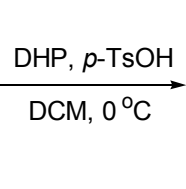<smiles>O=C1C[C@H]2CC(CC[C@H]([OH2+])CCc3ccccc3)C(=O)C[C@H]2O1</smiles>

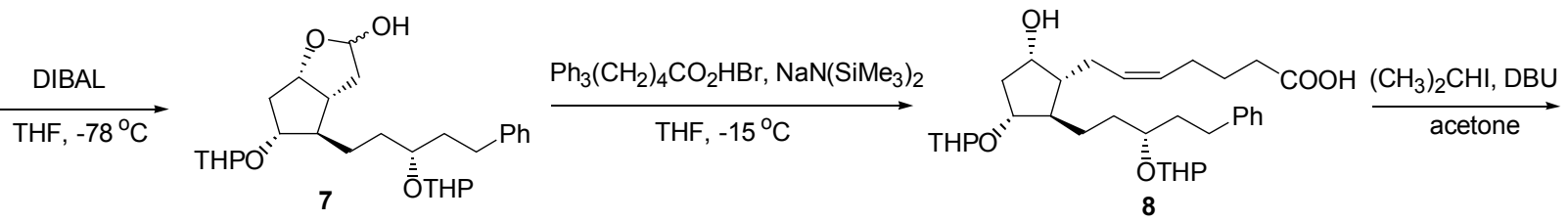

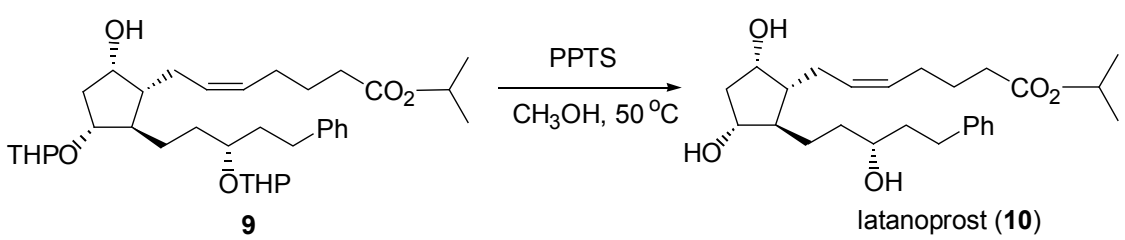

Scheme 2 


\section{1 结果与讨论}

\section{1 合成策略及方法的选择}

以科里内酯为起始原料的合成策略及方法中, Resul 等 ${ }^{[22]}$ 报道的合成路线(Scheme 3) 是比较常用的方法, 该 路线是以苯基苯甲酰基科里内酯为原料, 内酯氧化成醛 后，通过改良 Horner-Wadsworth-Emmons (HWE) 反应形 成 $\omega$ 侧链, 还原 $\omega$ 侧链、氢化、脱保护、内酯还原, Wittig 反应形成 $\alpha$ 侧链, 然后经酯化反应, 合成了拉坦前列素. 该合成路线步骤较少(8步), 但立体选择性差, 且内酯还 原过程中缺少保护基，导致收率较低(5.0\%).

经深入研究和分析, 我们发现该路线在内酯氧化成 醛、HWE 反应、还原 $\omega$ 侧链、氢化、内酯还原、Wittig 反应等步骤均存在一定的问题. 为了有效解决这些问 题, 我们选择了商品化的苯甲酰基科里内酯为原料, 以 (一)-DIP-Cl 为手性还原剂, 以 THP 为保护基, 设计了一 个 10 步反应路线(如 Scheme 2 所示), 并且成功地合成 了拉坦前列素，收率达 $23.5 \%$. 本文设计的合成路线与 原方法相比较, 具有较多优势, 具体如下: 在内酯氧化 成醛的反应步骤中，原合成方法使用 $\mathrm{DCC} /$ 磷酸氧化 剂, 氧化效率低; 本研究选用 Dess-Martin Oxidation 作 氧化剂，选择性好，氧化效率高，反应后处理简单易行.

在改良的 HWE 反应步骤中，原合成方法使用氢化 钠做催化剂, 导致少量保护基的水解, 前两步收率只有 $58.5 \%$; 而本研究选用氯化锂作催化剂, 反应条件温和、 效率高，前两步收率达到 $80.5 \%$.

在还原 $\omega$ 侧链的反应步骤中, 原合成方法使用氢化 铝锂作还原剂, 在一 $130{ }^{\circ} \mathrm{C}$ 反应，条件苛刻，立体选择 性差，不能控制对映异构体的生成，产率只有 52\% 而 生成大量的 $(R)$-异构体带入随后的反应中, 最终生成 $15(S)$ - 拉坦前列素异构体杂质; 本研究则选用 (一)-
DIP-Cl 作为手性还原剂，在一 $30{ }^{\circ} \mathrm{C}$ 反应，条件温和，立 体选择性较强，生成 $S$-异构体达 95\% 该步反应收率达 $74.3 \%$.

在氢化反应步骤中，原合成方法有少量 $\omega$ 侧链脱除 产物的生成; 而本研究使用乙醇作溶剂，同时加入少量 亚硝酸钠有效地防止了 $\omega$ 侧链脱除产物的生成.

在内酯还原为半缩醛的反应步骤中，原合成方法分 子中羟基未被保护，需要至少 4 倍量以上的 DIBAL，处 理困难，过高的用量导致内酯环的打开，生成 2 -\{(1R,2R,3R,5S)-3,5-二羟基-2-[(3R)-3-羟基-5-苯基戊 基]环戊基 $\}$ 乙酸，收率只有 $65 \%$; 本研究则使用 THP 作 保护基, DIBAL 只需 1.5 倍量，即可完全反应，同时避免 了副反应的发生，收率达 $92.3 \%$.

在 Wittig 反应步骤中，原合成方法使用非亲核性强 碱叔丁醇钾作催化剂形成磷叶立德，但使用叔丁醇钾, 需要 3 8 倍量的磷叶立德，且有 3\% 5\% 反式拉坦前列 素的形成，同时上一步产物中未保护的羟基影响了 Wittig 反应及随后酯化反应的效率，两步反应收率仅为 $38 \%$; 而本研究使用 NaHMDS 为催化剂形成磷叶立德, 只需要 2 倍量的磷叶立德，即可完全反应，且选择性好, 产物消旋体 $Z: E=99: 1$, 同时, 本研究用 THP 保护着 基后，也提高了 Wittig 反应和随后酯化反应的效率，两 步反应收率达 $77.8 \%$.

利用本研究合成路线方法合成拉坦前列素，最后收 率可以达到 $23.5 \%$ 远远高于原合成方法的收率(5.0\%); 另外本研究合成路线方法原料易得,反应条件温和，选 择性好，副反应少，收率高，因此更适合大批量合成.

\section{2 纯化方法的选择}

同以往合成方法，本研究合成方法制得的拉坦前列 素粗品，含有少量 15(S)-拉坦前列素(Impurity I)和 5,6-
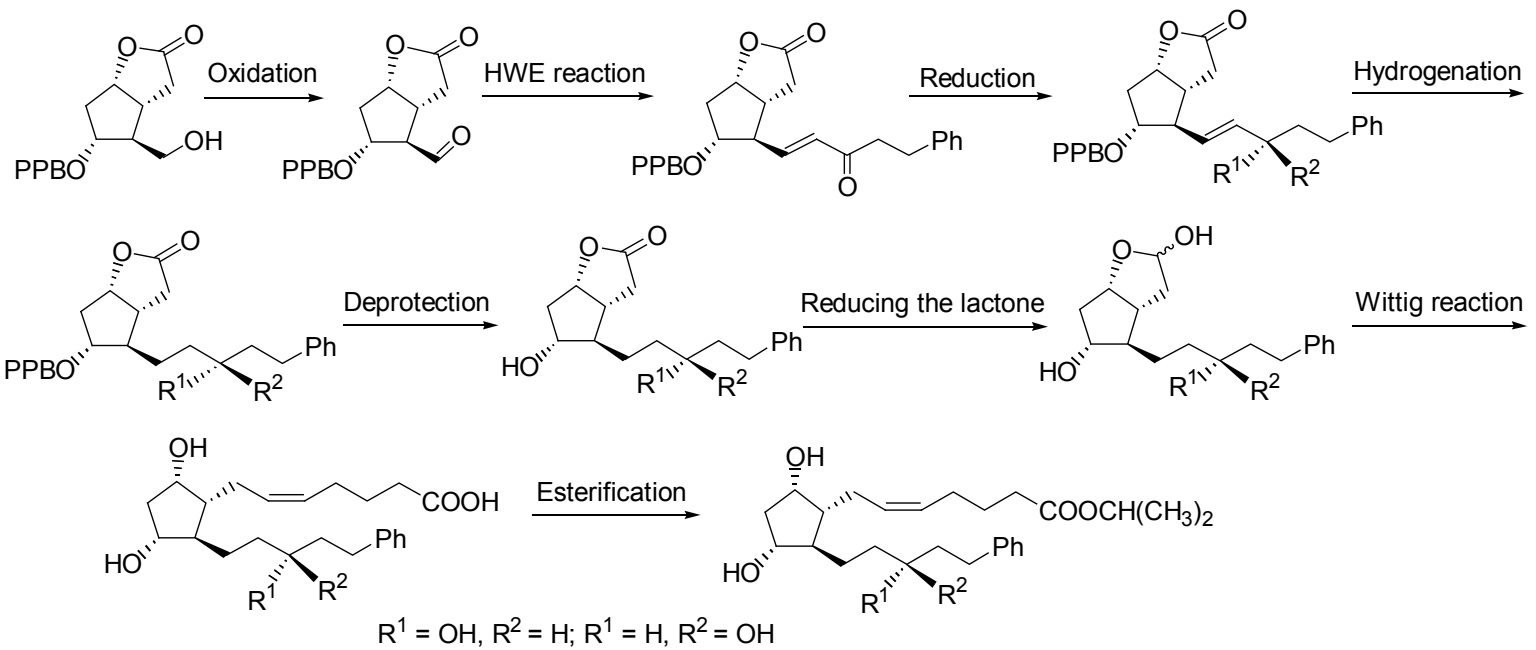

Scheme 3 
反式拉坦前列素(Impurity II)两种异构体杂质, 结构式 如 Scheme 4 所示.

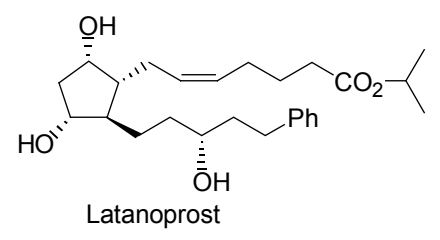

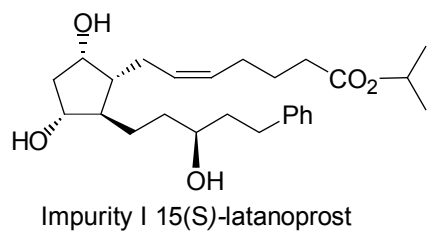<smiles>CC(C)C(=O)CCC/C=C\C[C@H]1C(O)C[C@@H](O)C1CCC[C@H](O)CCc1ccccc1</smiles>

Impurity II 5,6-trans-latanoprost

\section{Scheme 4}

以往纯化方法中均使用常压柱层析方法, 由于拉坦 前列素与两种异构体杂质物化性质相近, 极性差异较 小, 常压柱层析方法很难分离, 产物收率低、纯度低; 因 此, 本研究选用正相液相色谱法, 利用固定相的极性和 拉坦前列素与两种杂质极性差异, 通过在移动相中与固 定相相互作用力不同的差异, 将其实现分离. 经优化, 以 Waters SunFire $\mathrm{Si}$ 为固定相, 以正己烷/异丙醇/乙腈 为移动相, 且流动相组成为 $V$ (正己烷) : $V$ (异丙醇) : $V($ 乙腈 $)=92: 6.5: 1.5$ 时, 拉坦前列素和两种异构体杂 质因其极性差异和与硅胶表面上硅羟基作用力不同的 差异, 得到良好的分离, 纯化收率达到 $81.6 \%$. 拉坦前 列素纯化前粗品 HPLC 谱图见图 1(A), 经正相色谱法纯 化后所得纯品 HPLC 谱图见图 1(B).

可见, 纯化前粗品中两种异构体杂质保留时间分别 为 33.073 和 $41.342 \mathrm{~min}$, 与拉坦前列素的相对保留时间 分别为 0.92 和 1.15 ; 纯化后所得纯品经 HPLC 分析, 拉 坦前列素含量 $99.91 \%$ 5,6-反式拉坦前列素未检出, 15(S)-拉坦前列素含量 $0.09 \%$ 且杂质与拉坦前列素相对 保留时间一致, 见表 1 所示.

表 1 产物中拉坦前列素和异构体的相对保留时间和纯度

Table 1 Retention time and content of latanoprost and its isomers in product

\begin{tabular}{lcc}
\hline \multicolumn{1}{c}{ 产物成分 } & 相对保留时间 & 纯度(面积归一化法) \\
\hline 拉坦前列素 & 1.00 & $99.91 \%$ \\
$15(S)$-拉坦前列素 & 0.92 & $0.09 \%$ \\
5,6 -反式拉坦前列素 & 1.15 & 未检出 \\
\hline
\end{tabular}
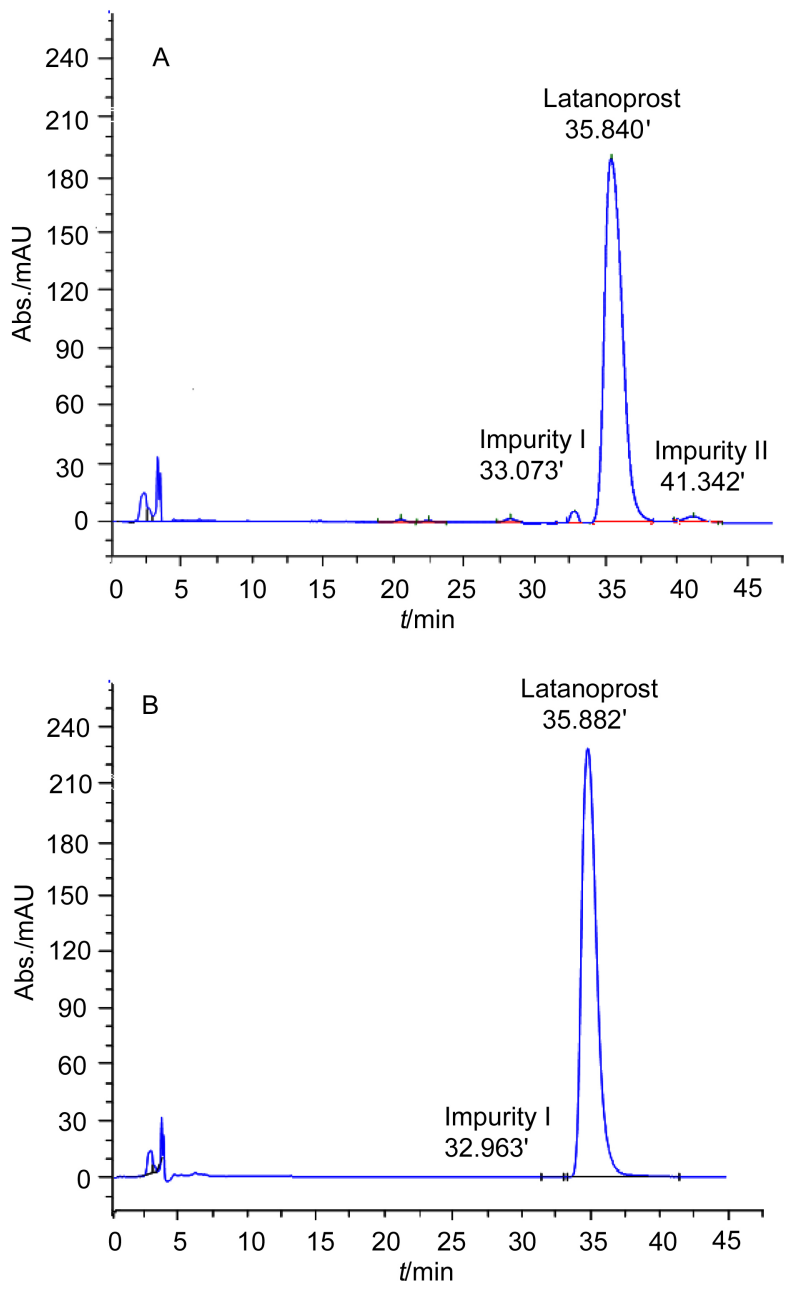

图 1 拉坦前列素粗品(A)和纯品(B)液相色谱图

Figure 1 HPLC of latanoprost crude (A) and puirity (B)

\section{2 结论}

本研究以苯甲酰基科里内酯为原料, 通过 10 步反 应完成拉坦前列素的合成, 用正相液相色谱法加以纯 化, 产物用 ${ }^{1} \mathrm{H}$ NMR, ${ }^{13} \mathrm{C}$ NMR, IR 和 HRMS 进行了结 构表征, 结果表明, 拉坦前列素与杂质得到良好的分离, 纯度达 $99.91 \%$ 总收率为 $19.2 \%$. 与以往合成方法相比, 本研究方法原料易得, 合成步骤少, 副反应少, 产物纯 度高, 收率高, 可大批量生产. 本研究为合成拉坦前列 素提供了一种新方法.

\section{3 实验部分}

\section{1 仪器与试剂}

液相色谱仪(LC-2010AHT); 核磁共振仪(BRUKER AV400); 高分辨质谱(Waters Q-TofMS/MS); 红外色谱 仪(BRUKER Vertex 70 FTIR); 高压制备液相系统(ETN P1000; DAC-50; ETN UV200). 所用原料和试剂均为国 
产, 反应溶剂均需无水干燥处理, 其中四氢呋喃采用钠/ 二苯甲酮体系干燥，二氯甲烷、丙酮用氢化钻干燥处理.

\section{2 实验方法}

3.2.1 (1S, $5 R, 6 R, 7 R)$-7-苯甲酰氧基-6-甲酰基-2-氧杂 双环[3.3.0]辛烷-3-酮(1)的合成

将 $55.3 \mathrm{~g}(0.2 \mathrm{~mol})$ 苯甲酰基科里内酯溶于 $280 \mathrm{~mL}$ 二氯甲烷， $0 \sim 5{ }^{\circ} \mathrm{C}$, 缓慢加入到 $127.2 \mathrm{~g}(0.3 \mathrm{~mol})$ Dess-Martin $/ 650 \mathrm{~mL}$ 二氯甲烷悬浮液中, 室温反应 $3 \mathrm{~h}$. 加入 $50 \mathrm{~g}$ 碳酸氢钠和 $110 \mathrm{~g}$ 硫代硫酸钠 $/ 600 \mathrm{~mL}$ 水的溶 液充分摚拌 $30 \mathrm{~min}$, 分液, 有机层用饱和氯化钠溶液洗 涤, 无水硫酸钠干燥, 得化合物 $\mathbf{1}$ 溶液. TLC $R_{\mathrm{f}}=0.8$ ( 乙 酸 乙酯); HRMS calcd for $\mathrm{C}_{15} \mathrm{H}_{14} \mathrm{NaO}_{5}[\mathrm{M}+\mathrm{Na}]^{+}$ 297.0739, found 297.0742.

3.2.2 (1S, $5 R, 6 R, 7 R)$-7- 苯甲酰氧基-6-[3-氧代-5-苯 基-(E)-1-戊烯基]-2-氧杂双环 [3.3.0]辛烷-3-酮(2)的合 成

氮气保护下, 将 $9.3 \mathrm{~g}(0.22 \mathrm{~mol})$ 氯化锂加入 $400 \mathrm{~mL}$ 四氢呋喃中, 降温至 $-5{ }^{\circ} \mathrm{C}$, 缓慢加入 $52.7 \mathrm{~g}(0.21 \mathrm{~mol})$ 2 -氧代-4-苯丁基膦酸二甲酯 $/ 240 \mathrm{~mL}$ 四氢呋喃, $0{ }^{\circ} \mathrm{C}$ 下 搅拌反应 $1 \mathrm{~h}$, 然后降温至 $-10{ }^{\circ} \mathrm{C}$, 缓慢加入化合物 $\mathbf{1}$, 继续反应 $1 \mathrm{~h}$. 加入 $1 \mathrm{moL}$ 盐酸调 $\mathrm{pH}$ 至中性, 分液, 用 饱和氯化钠溶液洗涤, 无水硫酸钠干燥, 浓缩至干, 用 乙醚重结晶, 得白色粉末 $65.2 \mathrm{~g}$, 产率 $80.5 \%$. m.p. $117 \sim 118{ }^{\circ} \mathrm{C},[\alpha]_{\mathrm{D}}^{20}-116.3\left(c=1.0, \mathrm{CH}_{3} \mathrm{CN}\right)$, TLC $R_{\mathrm{f}}=$ 0.7 [ $V($ 正己烷 $): V($ 乙酸乙酯 $)=1: 1$ ] ; ${ }^{1} \mathrm{H}$ NMR $(400$ $\left.\mathrm{MHz}, \mathrm{CDCl}_{3}\right) \delta: 2.29(\mathrm{dd}, J=4.8,15.6 \mathrm{~Hz}, 1 \mathrm{H}), 2.48(\mathrm{~d}$, $J=16.8 \mathrm{~Hz}, 1 \mathrm{H}), 2.57 \sim 2.62(\mathrm{~m}, 1 \mathrm{H}), 2.85 \sim 2.95(\mathrm{~m}, 7 \mathrm{H})$, $5.06 \sim 5.09(\mathrm{~m}, 1 \mathrm{H}), 5.28(\mathrm{dd}, J=5.2,11.2 \mathrm{~Hz}, 1 \mathrm{H}), 6.20$ $(\mathrm{d}, J=15.6 \mathrm{~Hz}, 1 \mathrm{H}), 6.65(\mathrm{dd}, J=7.8,16 \mathrm{~Hz}, 1 \mathrm{H}), 7.17 \sim$ 7.20 (m, 3H), 7.27 (t, $J=7.6 \mathrm{~Hz}, 2 \mathrm{H}), 7.45(\mathrm{t}, J=7.6 \mathrm{~Hz}$, $2 \mathrm{H}), 7.58(\mathrm{t}, J=7.6 \mathrm{~Hz}, 1 \mathrm{H}), 7.98(\mathrm{~d}, J=7.6 \mathrm{~Hz}, 2 \mathrm{H})$; HRMS calcd for $\mathrm{C}_{25} \mathrm{H}_{24} \mathrm{NaO}_{5}[\mathrm{M}+\mathrm{Na}]^{+}$427.1521, found 427.1526.

3.2.3 ( $1 S, 5 R, 6 R, 7 R)$-7 - 苯甲酰氧基-6-[3(S)-3-羟基-5苯基-1(E)-戊烯基]-2-氧杂双环[3.3.0]辛烷-3-酮 (3) 的 合成

氮气保护下, 将 $60.7 \mathrm{~g}(0.15 \mathrm{~mol})$ 化合物 2 加入 660 $\mathrm{mL}$ 四氢呋喃, 控温 $-30{ }^{\circ} \mathrm{C}$ 下滴加 $192.5 \mathrm{~g}$ (0.6 mol) (一)-二异松蒎基氯硼烷 $390 \mathrm{~mL}$ 四氢呋喃溶液, 滴加完 毕后控温反应 $3 \mathrm{~h}$. 加入 $400 \mathrm{~mL}$ 甲醇和 $200 \mathrm{~mL}$ 丙酮, 室 温搅拌 $1 \mathrm{~h}$, 浓缩, 加入 $1500 \mathrm{~mL}$ 二氯甲烷, 加入 $5 \%$ 碳 酸氢钠溶液 $800 \mathrm{~mL}$, 分液, 有机层用饱和氯化钠溶液洗 涤, 用无水硫酸钠干燥, 浓缩, 硅胶柱层析 $[V($ 正己 烷) : $V$ (乙酸乙酯 $)=1: 1$ ], 接收组份, 浓缩至干, 用乙 醚重结晶, 得白色粉末 $45.3 \mathrm{~g}$, 收率 74.3\%. m.p. 79 81
${ }^{\circ} \mathrm{C},[\alpha]_{\mathrm{D}}^{20}-103.0\left(c=1.0, \mathrm{CH}_{3} \mathrm{CN}\right)$, TLC $R_{\mathrm{f}}=0.5$ [V(正己 烷 $): V($ 乙酸乙酯 $)=1: 1] ;{ }^{1} \mathrm{H}$ NMR $\left(400 \mathrm{MHz}, \mathrm{CDCl}_{3}\right)$ $\delta: 1.76 \sim 1.87(\mathrm{~m}, 2 \mathrm{H}), 2.20 \sim 2.26(\mathrm{~m}, 1 \mathrm{H}), 2.50(\mathrm{~d}, J=$ $17.2 \mathrm{~Hz}, 1 \mathrm{H}), 2.57 \sim 2.87(\mathrm{~m}, 7 \mathrm{H}), 4.13(\mathrm{q}, J=6 \mathrm{~Hz}, 1 \mathrm{H})$, $5.03 \sim 5.07(\mathrm{~m}, 1 \mathrm{H}), 5.25(\mathrm{q}, J=6 \mathrm{~Hz}, 1 \mathrm{H}), 5.57 \sim 5.71(\mathrm{~m}$, $2 \mathrm{H}), 7.13 \sim 7.16(\mathrm{~m}, 2 \mathrm{H}), 7.19(\mathrm{~d}, J=7.6 \mathrm{~Hz}, 1 \mathrm{H}), 7.24 \sim$ $7.28(\mathrm{~m}, 2 \mathrm{H}), 7.43(\mathrm{t}, J=8.0 \mathrm{~Hz}, 2 \mathrm{H}), 7.56$ (t, $J=7.2 \mathrm{~Hz}$, $1 \mathrm{H}), 7.98(\mathrm{~d}, J=7.2 \mathrm{~Hz}, 2 \mathrm{H})$; HRMS calcd for $\mathrm{C}_{25} \mathrm{H}_{26} \mathrm{Na}$ $\mathrm{O}_{5}[\mathrm{M}+\mathrm{Na}]^{+} 429.1678$, found 429.1677 .

3.2.4 (1S,5R,6R,7R)-7-羟基-6-[3(S)-3-羟基-5-苯基$1(E)$-戊烯基]-2-氧杂双环 [3.3.0]辛烷-3-酮(4)的合成

将 $44.7 \mathrm{~g}(0.11 \mathrm{~mol})$ 化合物 3 加入 $450 \mathrm{~mL}$ 甲醇中, 降温至 $0{ }^{\circ} \mathrm{C}$, 加入 $9.1 \mathrm{~g}(0.066 \mathrm{~mol})$ 碳酸钾, 然后室温反 应 $3 \mathrm{~h}$. 加入用 $1 \mathrm{moL}$ 盐酸调 $\mathrm{pH}$ 至中性, 用乙酸乙酯萃 取 3 次, 每次 $500 \mathrm{~mL}$, 有机层用饱和氯化钠溶液洗涤, 无水硫酸钠干燥, 过滤, 浓缩, 硅胶柱层析(乙酸乙酯), 收集组份, 浓缩, 干燥, 得无色油状液体 $29.4 \mathrm{~g}$, 收率 $88.5 \%$. $[\alpha]_{\mathrm{D}}^{20}-31.1\left(c=1.0, \mathrm{CH}_{3} \mathrm{CN}\right)$, TLC $R_{\mathrm{f}}=0.1$ $[V($ 正己烷 $): V($ 乙酸乙酯 $)=1: 1] ;{ }^{1} \mathrm{H}$ NMR $(400 \mathrm{MHz}$, $\left.\mathrm{CDCl}_{3}\right) \delta: 1.83 \sim 1.94(\mathrm{~m}, 4 \mathrm{H}), 2.46 \sim 2.74(\mathrm{~m}, 7 \mathrm{H})$, $3.94 \sim 3.97(\mathrm{q}, J=7.2 \mathrm{~Hz}, 1 \mathrm{H}), 4.05 \sim 4.15(\mathrm{~m}, 2 \mathrm{H}) 4.88 \sim$ $4.91(\mathrm{~m}, 1 \mathrm{H}), 5.57(\mathrm{ddd}, J=6.4,15.6,66.8 \mathrm{~Hz}, 2 \mathrm{H})$, $7.18 \sim 7.21(\mathrm{~m}, 3 \mathrm{H}), 7.27 \sim 7.31(\mathrm{~m}, 2 \mathrm{H})$; HRMS calcd for $\mathrm{C}_{18} \mathrm{H}_{23} \mathrm{O}_{4}[\mathrm{M}+\mathrm{H}]^{+}$303.1596, found 303.1599.

3.2.5 ( $1 S, 5 R, 6 R, 7 R)$-7-羟基-6-[3(R)-3-羟基-5-苯基$1(E)$-戊基]-2-氧杂双环 [3.3.0]辛烷-3-酮(5)的合成

将 $27.2 \mathrm{~g}(0.09 \mathrm{~mol})$ 化合物 4 溶于 $600 \mathrm{~mL}$ 无水乙醇 中, 加入 $1.0 \mathrm{~g}(0.015 \mathrm{~mol})$ 亚硝酸钠、 $1.6 \mathrm{~g}$ 钯/碳催化剂, 通入氢气，于 $5 \mathrm{MPa}$ 下室温反应 $5 \mathrm{~h}$. 过滤，浓缩，加入 $0.1 \mathrm{moL}$ 盐酸 $150 \mathrm{~mL}$, 搅拌 $10 \mathrm{~min}$, 用乙酸乙酯萃取 3 次, 每次 $400 \mathrm{~mL}$, 合并有机层, 饱和氯化钠溶液洗涤, 无水硫酸钠干燥, 浓缩, 得无色油状液体 $23.3 \mathrm{~g}$, 收率 85.2\%. $[\alpha]_{\mathrm{D}}^{20}-17.8\left(c=1.0, \mathrm{CH}_{3} \mathrm{CN}\right), \mathrm{TLC} R_{\mathrm{f}}=0.5$ (乙酸 乙酯); ${ }^{1} \mathrm{H}$ NMR ( $\left.400 \mathrm{MHz}, \mathrm{CDCl}_{3}\right) \delta: 1.48 \sim 1.57(\mathrm{~m}, 3 \mathrm{H})$, $1.73 \sim 1.81(\mathrm{~m}, 3 \mathrm{H}), 2.24 \sim 2.31(\mathrm{~m}, 2 \mathrm{H}), 2.47 \sim 2.54(\mathrm{~m}$, $3 \mathrm{H}), 2.63 \sim 2.69(\mathrm{~m}, 1 \mathrm{H}), 2.76 \sim 2.79(\mathrm{~m}, 2 \mathrm{H}), 3.68$ (br., $1 \mathrm{H}), 3.72(\mathrm{q}, J=8.0 \mathrm{~Hz}, 2 \mathrm{H}), 4.12(\mathrm{q}, J=8.0 \mathrm{~Hz}, 1 \mathrm{H})$, $4.90 \sim 4.94(\mathrm{~m}, 1 \mathrm{H}), 7.19$ (d, $J=8.0 \mathrm{~Hz}, 3 \mathrm{H}), 7.28$ (t, $J=$ $8.0 \mathrm{~Hz}, 2 \mathrm{H})$; HRMS calcd for $\mathrm{C}_{18} \mathrm{H}_{25} \mathrm{O}_{4}[\mathrm{M}+\mathrm{H}]^{+}$ 305.1753 , found 305.1758 .

3.2.6 ( $1 S, 5 R, 6 R, 7 R)$-7-羟基-6- $[3(R)$-3-羟基-(5-苯基3-四氢- $2 \mathrm{H}$-吡喃-2-基氧基)戊基]-7-四氢- $2 \mathrm{H}$-吡喃-2基氧基-2-氧杂双环 [3.3.0]辛烷-3-酮(6)的合成

将 $21.3 \mathrm{~g}(0.07 \mathrm{~mol})$ 化合物 $\mathbf{5}$ 加入 $500 \mathrm{~mL}$ 二氯甲烷 中溶解, 加入 $17.7 \mathrm{~g}(0.21 \mathrm{~mol}) 3,4$-二氢吡喃 $/ 60 \mathrm{~mL}$ 四氢 
呋喃, 降温至 $0{ }^{\circ} \mathrm{C}$, 加入 $0.27 \mathrm{~g}(1.4 \mathrm{mmol})$ 对甲苯磺酸, 控温 0 反应 $3 \mathrm{~h}$. 加入 $5 \%$ 碳酸氢钠 $300 \mathrm{~mL}$ 搅拌 $1 \mathrm{~h}$, 分 液, 有机层用饱和氯化钠溶液洗涤, 无水硫酸钠干燥, 浓缩, 得淡黄色油状液体 $28.3 \mathrm{~g}$, 收率 $85.6 \%$. [ $\alpha]_{\mathrm{D}}^{20}-$ $13.5\left(c=1.0, \mathrm{CH}_{3} \mathrm{CN}\right), \mathrm{TLC} R_{\mathrm{f}}=0.8$ [ $V$ (正己烷)：V(乙酸 乙酯 $)=1: 1] ;{ }^{1} \mathrm{H} \mathrm{NMR}\left(400 \mathrm{MHz}, \mathrm{CDCl}_{3}\right) \delta: 1.24 \sim 2.01$ (m, $20 \mathrm{H}), 2.15 \sim 2.25(\mathrm{~m}, 1 \mathrm{H}), 2.42 \sim 2.67(\mathrm{~m}, 3 \mathrm{H}), 2.74 \sim$ $2.82(\mathrm{~m}, 2 \mathrm{H}), 3.44 \sim 3.51(\mathrm{~m}, 2 \mathrm{H}), 3.64 \sim 3.69(\mathrm{~m}, 1 \mathrm{H})$, $3.79 \sim 3.94(\mathrm{~m}, 3 \mathrm{H}), 4.57 \sim 4.68(\mathrm{~m}, 2 \mathrm{H}), 4.95 \sim 5.00(\mathrm{~m}$, $1 \mathrm{H}), 7.16 \sim 7.21(\mathrm{~m}, 3 \mathrm{H}), 7.26 \sim 7.29(\mathrm{~m}, 2 \mathrm{H})$; IR $(\mathrm{KBr}) v$ : 2941, 2867, 1774, 1603, 1496, 1454, 1353, 1200, 1175, $1133,1076,1024,748,701 \mathrm{~cm}^{-1}$; HRMS calcd for $\mathrm{C}_{28} \mathrm{H}_{40^{-}}$ $\mathrm{NaO}_{6}[\mathrm{M}+\mathrm{Na}]^{+}$495.2723, found 495.2727

3.2.7 ( $1 S, 5 R, 6 R, 7 R)-6$ - $[3(R)-(5$ - 苯基-3-四氢 $-2 H$ - 吡 喃-2-基氧基)戊基]-7-四氢- $2 \mathrm{H}$-吡喃-2-基氧基-2-氧杂 双环 [3.3.0]辛烷-3-醇(7)的合成

将 $28.3 \mathrm{~g}(0.06 \mathrm{~mol})$ 化合物 $\mathbf{6}$ 溶于 $600 \mathrm{~mL}$ 四氢呋喃, 氮气保护下, 控温 $-78{ }^{\circ} \mathrm{C}$ 缓慢滴加入 $0.09 \mathrm{~mol}$ 二异丁 基氢化铝/正已烷溶液( $1.0 \mathrm{~mol} / \mathrm{L}$ ), 控温反应 $1 \mathrm{~h}$. 恢复到 室温, 加入甲醇 $200 \mathrm{~mL}$, 摚拌 $10 \mathrm{~min}$, 浓缩, $0.1 \mathrm{~mol} / \mathrm{L}$ 盐酸调 $\mathrm{pH}$ 至中性, 加入水 $300 \mathrm{~mL}$, 用乙酸乙酯萃取 3 次, 每次 $500 \mathrm{~mL}$, 合并有机层, 用饱和氯化钠溶液洗涤, 无水硫酸钠干燥, 浓缩, 得无色油状液体 $26.2 \mathrm{~g}$, 收率 $92.3 \% . \quad[\alpha]_{\mathrm{D}}^{20}-19.0\left(c=1.0, \mathrm{CH}_{3} \mathrm{CN}\right)$, TLC $R_{\mathrm{f}}=0.6$ $[V($ 正己烷 $): V($ 乙酸乙酯 $)=1: 1] ;{ }^{1} \mathrm{H}$ NMR $(400 \mathrm{MHz}$, $\left.\mathrm{CDCl}_{3}\right) \delta: 1.22 \sim 1.29(\mathrm{~m}, 1 \mathrm{H}), 1.54 \sim 2.38(\mathrm{~m}, 23 \mathrm{H})$, $2.60 \sim 2.68(\mathrm{~m}, 2 \mathrm{H}), 2.77 \sim 2.79(\mathrm{~m}, 1 \mathrm{H}), 3.46 \sim 3.52(\mathrm{~m}$, $2 \mathrm{H}), 3.67 \sim 3.74(\mathrm{~m}, 2 \mathrm{H}), 3.85 \sim 3.97(\mathrm{~m}, 3 \mathrm{H}), 4.61 \sim 4.65$ (m, $2 \mathrm{H}), 5.42 \sim 5.62(\mathrm{~m}, 1 \mathrm{H}), 7.16 \sim 7.21(\mathrm{~m}, 3 \mathrm{H}), 7.25 \sim$ 7.29 (m, 2H); IR (KBr) v: 3417, 2940, 2867, 1720, 1603, 1496, 1454, 1353, 1201, 1133, 1076, 1023, 746, $700 \mathrm{~cm}^{-1}$; HRMS calcd for $\mathrm{C}_{28} \mathrm{H}_{42} \mathrm{NaO}_{6}[\mathrm{M}+\mathrm{Na}]^{+} 497.2879$, found 497.2883.

3.2 .8 ( $Z$ )- $7-\{(1 R, 2 R, 3 R, 5 S)-5$ - 羟基-2-[3(R)-(5-苯基3 -四氢- $2 H$-吡喃-2-基氧基)戊基]-3-(四氢- $2 H$-吡喃-2基氧基)环戊基 $\}-5$-庚烯酸 (8)的合成

将(4-羧基丁基)三苯基澳化鏻 $44.3 \mathrm{~g}(0.10 \mathrm{~mol})$ 加入 $500 \mathrm{~mL}$ 四氢呋喃中, 氩气保护下, 降温至 $-15{ }^{\circ} \mathrm{C}$, 加 入 $100 \mathrm{~mL}(0.2 \mathrm{~mol})$ 六甲基二硅基胺基钠 $(2 \mathrm{~mol} / \mathrm{L})$, 摚 拌反应 $1 \mathrm{~h}$, 加入 $23.7 \mathrm{~g}(0.05 \mathrm{~mol})$ 化合物 7/60 mL 四氢 呋喃溶液, 反应 $2 \mathrm{~h}$. 加入冰 $30 \mathrm{~g}$, 搅拌 $10 \mathrm{~min}$, 用 1 $\mathrm{mol} / \mathrm{L}$ 盐酸调 $\mathrm{pH}$ 至中性, 加入 $600 \mathrm{~mL}$ 二氯甲烷萃取, 饱和氯化钠溶液洗涤, 无水硫酸钠干燥, 浓缩后得产物. TLC $R_{\mathrm{f}}=0.4$ [ $V$ (正已烷) $: V($ 乙酸乙酯 $): V($ 冰乙酸 $)=$ $10: 5: 0.5]$; HRMS calcd for $\mathrm{C}_{33} \mathrm{H}_{50} \mathrm{NaO}_{7}[\mathrm{M}+\mathrm{Na}]^{+}$
581.3454 , found 581.3459 .

$3.2 .9(Z)-7-\{(1 R, 2 R, 3 R, 5 S)-5$-羟基- 2 -[ $[3(R)$ - ( 5 - 苯基3 -四氢- $2 H$-吡喃-2-基氧基)戊基]-3-(四氢- $2 H$-吡喃-2基氧基)环戊基 $\}-5$-庚烯酸异丙酯(9)的合成

将化合物 8 溶于 $1200 \mathrm{~mL}$ 丙酮, $0{ }^{\circ} \mathrm{C}$ 下, 滴加入 15.2 $\mathrm{g}(0.1 \mathrm{~mol}) \mathrm{DBU}$, 升到室温后, 加入 $17.0 \mathrm{~g}(0.1 \mathrm{~mol}) 2-$ 碘丙烷, 室温反应约 $24 \mathrm{~h}$. 将反应液浓缩, 加入 $3 \%$ 柠檬 酸调 $\mathrm{pH}$ 至中性, 加入 $1000 \mathrm{~mL}$ 二氯甲烷, 分液, 有机层 用饱和盐水洗涤, 无水硫酸钠干燥, 浓缩, 硅胶柱层析, 用 $V$ (正已烷 $): V($ 乙酸乙酯 $)=2: 1$ 洗脱, 接收主峰, 浓 缩得无色油状液体 $23.4 \mathrm{~g}$, 收率 $77.8 \%$; $[\alpha]_{\mathrm{D}}^{20}+76.5$ $\left(c=1.0, \mathrm{CH}_{3} \mathrm{CN}\right)$, TLC $R_{\mathrm{f}}=1.0$ [ $V($ 正己烷 $): V($ 乙酸乙 酯 $): V($ 冰乙酸 $)=10: 5: 0.5] ;{ }^{1} \mathrm{H}$ NMR $(400 \mathrm{MHz}$, $\left.\mathrm{CDCl}_{3}\right) \delta: 1.22(\mathrm{~d}, J=7.2 \mathrm{~Hz}, 6 \mathrm{H}), 1.32 \sim 1.89(\mathrm{~m}, 23 \mathrm{H})$, $2.13 \sim 2.66(\mathrm{~m}, 9 \mathrm{H}), 3.49 \sim 3.50(\mathrm{~m}, 2 \mathrm{H}), 3.66 \sim 4.07(\mathrm{~m}$, $6 \mathrm{H}), 4.64 \sim 4.68(\mathrm{~m}, 2 \mathrm{H}), 4.96 \sim 5.03(\mathrm{~m}, 1 \mathrm{H}), 5.37 \sim 5.52$ (m, 2H), $7.17 \sim 7.29$ (m, 5H); IR (KBr) v: 2927, 2869, 1730, 1455, 1376, 1111, 1023, 746, $700 \mathrm{~cm}^{-1}$; HRMS calcd for $\mathrm{C}_{36} \mathrm{H}_{56} \mathrm{NaO}_{7}[\mathrm{M}+\mathrm{Na}]^{+}$623.3924, found 623.3922 .

3.2.10 (Z)-7- $\{(1 R, 2 R, 3 R, 5 S)-3,5-$ 二羟基 - $2-[3(R)-(3-$ 羟基-5-苯基)戊基]环戊基 $\}-5$-庚烯酸异丙酯 (10, latanoprost)的合成

将 $18.0 \mathrm{~g}(0.03 \mathrm{~mol})$ 化合物 9 加入 $500 \mathrm{~mL}$ 甲醇, 加 入 $0.4 \mathrm{~g}(0.0015 \mathrm{~mol})$ 对甲苯磺酸吡啶, 摚拌, 控温 $50{ }^{\circ} \mathrm{C}$ 反应 $4 \mathrm{~h}$. 浓缩, 加入 $5 \%$ 碳酸氢钠调 $\mathrm{pH}$ 至中性, 加入 $500 \mathrm{~mL}$ 乙酸乙酯, 分液, 有机层用饱和氯化钠溶液洗 涤, 无水硫酸钠干燥, 浓缩得产物约 $12.5 \mathrm{~g}$. 硅胶柱层 析用 $V$ (正已烷 $): V($ 乙酸乙酯 $)=1: 1$ 洗脱, 分段接收, 用液相色谱检测纯度[HPLC, 检测波长: $210 \mathrm{~nm}$, 色谱 柱: Waters SunFire Si $(5 \mu \mathrm{m}, 4.6 \mathrm{~mm} \times 250 \mathrm{~mm})$, 流动相: $V($ 正己烷 $): V$ (异丙醇) $: V($ 乙腈 $)=93: 6: 1$, 柱温 35 ${ }^{\circ} \mathrm{C}$, 流速 $1.0 \mathrm{~mL} / \mathrm{min}$, 下同], 合并纯度 $95 \%$ 以上主峰, 浓 缩, 得无色油状液体 $10.9 \mathrm{~g}$, 收率 $84.7 \%$.

\subsubsection{1 拉坦前列素的纯化}

将 $10.9 \mathrm{~g}$ 粗品经高压制备液相系统(制备色谱柱 [DAC-50, Waters SunFire Si $(10 \mu \mathrm{m}, 50 \mathrm{~mm} \times 650 \mathrm{~mm})$; 波长 $210 \mathrm{~nm}$, 流速 $50 \mathrm{~mL} / \mathrm{min}$, 流动相: $V$ (正己烷)：V(异 丙醇 $): V($ 乙腈 $)=92: 6.5: 1.5$, 下同]多次上样, 接收 主峰, 合并, 浓缩, 真空干燥, 得无色油状液体 $8.9 \mathrm{~g}$, 收率 $81.6 \%$. 纯度为 $99.91 \% .[\alpha]_{\mathrm{D}}^{20}+35.0(c=1.0$, $\left.\mathrm{CH}_{3} \mathrm{CN}\right), \mathrm{TLC} R_{\mathrm{f}}=0.2$ [ $V($ 正己烷 $): V$ (乙酸乙酯 $)=$ $2: 1]$; ${ }^{1} \mathrm{H}$ NMR $\left(400 \mathrm{MHz}, \mathrm{CDCl}_{3}\right) \delta: 1.22(\mathrm{~d}, J=6.0 \mathrm{~Hz}$, $6 \mathrm{H}), 1.25 \sim 1.39(\mathrm{~m}, 2 \mathrm{H}), 1.49 \sim 1.80(\mathrm{~m}, 9 \mathrm{H}), 1.81 \sim 1.87$ (m, $2 \mathrm{H}), 2.11 \sim 2.14(\mathrm{~m}, 2 \mathrm{H}), 2.26 \sim 2.33(\mathrm{~m}, 5 \mathrm{H}), 2.46$ 
(brs, $1 \mathrm{H}), 2.65 \sim 2.71(\mathrm{~m}, 1 \mathrm{H}), 2.76 \sim 2.80(\mathrm{~m}, 1 \mathrm{H}), 3.66$ (quint, $J=5.6 \mathrm{~Hz}, 1 \mathrm{H}), 3.94(\mathrm{br}, 1 \mathrm{H}), 4.16(\mathrm{br}, 1 \mathrm{H}), 5.00$ (quint, $J=6.4 \mathrm{~Hz}, 1 \mathrm{H}), 5.36 \sim 5.49(\mathrm{~m}, 2 \mathrm{H}), 7.16 \sim 7.21$ $(\mathrm{m}, 3 \mathrm{H}), 7.26 \sim 7.30(\mathrm{~m}, 2 \mathrm{H}) ;{ }^{13} \mathrm{C}$ NMR $(100 \mathrm{MHz}$, $\left.\mathrm{CDCl}_{3}\right) \delta: 21.82,24.91,26.61,26.90,29.63,32.10,34.03$, $35.79,39.04,42.49,51.88,52.88,67.64,71.29,74.69$, $78.78,125.80,128.38,129.32,129.58,142.07,173.47$; IR (KBr) v: 3397, 3085, 3062, 3025, 2933, 1729, 1496, 1455, $1109,1031,996,747,700 \mathrm{~cm}^{-1}$; HRMS calcd for $\mathrm{C}_{26} \mathrm{H}_{40^{-}}$ $\mathrm{NaO}_{5}[\mathrm{M}+\mathrm{Na}]^{+}$455.2773, found 455.2777.

\section{References}

[1] Straus, D. S.; Christopher, K. Med. Res. Rev. 2001, 21, 185.

[2] Allaj, V.; Guo, C.; Nie, D. Cell Biosci. 2013, 8, 1.

[3] Selliah, R. D.; Hellberg, M. R.; Sharif, N. A.; McLaughlin, M. A. Bioorg. Med. Chem. Lett. 2004, 14, 4525.

[4] Han, Z. B.; Chang, Y. P.; Luan, G. Y.; Li, J.; Wang, E. B. Chem. J. Chin. Univ. 2002, 23(9), 1660 (in Chinese). (韩正波, 常雅萍, 奕国有, 李娟, 王恩波, 高等学校化学学报, 2002, 23(9), 1660.)

[5] Liu, Z.-Y.; Han, W.-P. Acta Chim. Sinica 1981, 39(4), 424 (in Chinese)

(刘志煌, 韩卫平, 化学学报, 1981, 39(4), 424.)

[6] Das, S.; Chandrasekhar, S.; Yadav, J. S.; Gre, R. Chem. Rev. 2007, $107,3286$.

[7] Levkoeva, E. I.; Yakhontov, L. N. Russ. Chem. Rev. 1977, 46, 565.

[8] Collins, P. W.; Djuric, S. W. Chem. Rev. 1999, 93, 1533.

[9] Ying, Y.; Lucitt, M. B.; Stubbe, J.; Yan, C.; Friis, U. G.; Hansen, P.
B.; Jensen, B. L.; Smyth, E. M.; FitzGerald, G. A. Proc. Natl. Acad. Sci. U. S. A. 2009, 106, 7985.

[10] Perrino, E.; Uliva, C.; Lanzi, C.; Soldato, P. D.; Masini, E.; Sparatore, A. Bioorg. Med. Chem. Lett. 2009, 19, 1639.

[11] DeLong, M. A.; Amburgey, J.; Taylor, C.; Wos, J. A.; Soper, D. L.; Wang, Y. L.; Hicks, R. Bioorg. Med. Chem. Lett. 2000, 10, 1519.

[12] Feng, Z. X.; Hellberg, M. R.; Sharif, N. A.; McLaughlin, M. A. Bioorg. Med. Chem. 2009, 17, 576.

[13] Allaire, C.; Dietrich, A.; Allmeier, H.; Grundmane, I.; Mazur, P. G.; Neshev, P.; Kahle, G. Eur. J. Ophthalmol. 2012, 22, 19.

[14] Steigerwalt, R. D.; Vingolo, E. M.; Plateroti, P.; Nebbioso, M. Eur. Rev. Med. Pharmacol. Sci. 2012, 16, 1723.

[15] Pacella, F.; Turchetti, P.; Santamaria, V.; Impallara, D.; Smaldone, G.; Brillante, C.; Librando, A.; Damiano, A.; Pecori, G. J.; Pacella, E. Clin. Ophthalmol. 2012, 6, 811 .

[16] Obadalova, I.; Pilarcik, T.; Slavikova, M.; Hajicek, J. Chirality 2005, 17, 109.

[17] Sharif, N. A. Prostaglandins, Leukotrienes Essent. Fatty Acids 2008, 78, 199.

[18] Klimko, P.; Hellberg, M.; McLaughlin, M.; Sharif, N. Bioorg. Med. Chem. 2004, 12, 3451.

[19] Liljebris, C.; Selen, G.; Resul, B.; Stjernschantz, J.; Hacksell, U. J. Med. Chem. 1996, 38, 289.

[20] Liu, Z.-Y.; Wu, X.-R. Chin. J. Org. Chem. 1981, 1, 104 (in Chinese). (刘志煌, 吴向荣, 有机化学, 1981, 1, 104.)

[21] Martynow, J. G.; wik, J. J.; Szelejewski, W.; Achmatowicz, O.; Kutner, A.; Wisniewski, K.; Winiarski, J.; Zegrocka, S. O.; biewski, P. G. Eur. J. Org. Chem. 2007, 689.

[22] Resul, B.; Stjernschantz, J.; No, K.; Liljebris, C.; SelBn, G.; Astin, M.; Karlsson, M.; Bib, L. Z. J. Med. Chem. 1993, 36, 243. 